\title{
Erratum to: The spin of prime ideals
}

\author{
J. B. Friedlander · H. Iwaniec • B. Mazur • \\ K. Rubin
}

Published online: 8 August 2015

(C) The Author(s) 2015

\section{Erratum to: Invent math (2013) 193:697-749 \\ DOI 10.1007/s00222-012-0438-8}

A certain argument concerning the coprimality of two variables turns out to be incorrect. We are grateful to Ruofan Wang for finding this error. On page 728 we claimed that $q$ and $m$ can be taken to be coprime. This is not always true but actually, in the application, we needed only that $q$ does not divide $m$. As a consequence, all of the results remain unchanged, except for a few modifications in the proofs. We make the following changes.

1. On page 723 , line 2 , replace $2^{2 h+3} f$ by $2^{2 h+3} f \mathfrak{D}$, where $\mathfrak{D}$ is the different.

The online version of the original article can be found under doi:10.1007/s00222-012-0438-8.

J. B. Friedlander $(\varangle)$

Department of Mathematics, University of Toronto, Toronto, ON M5S 2E4, Canada e-mail: frdlndr@math.toronto.edu

H. Iwaniec

Department of Mathematics, Rutgers University, New Brunswick, NJ 08903, USA

e-mail: iwaniec@math.rutgers.edu

B. Mazur

Department of Mathematics, Harvard University, Cambridge, MA 02138, USA

e-mail: mazur@math.harvard.edu

K. Rubin

Department of Mathematics, UC Irvine, Irvine, CA 92697, USA

e-mail: krubin@math.uci.edu 
2. On page 727 , replace the first two lines following (6.21) by

... where $\mathfrak{g}$ takes from $\mathfrak{c}$ all prime ideals of degree greater than one (recall that there are no ramified primes in $\mathfrak{c}$ which is coprime to $\mathfrak{D}$ ), and all primes of degree one for which some different ...

and three lines later, in the congruence, replace "mod $q$ " by "mod q".

3. On page 728 , replace the text starting from 'Finally, the congruence ...' and ending after (6.25), by

Finally, the congruence (6.12), together with $a \equiv a_{0}\left(\bmod g_{0}\right)$, means that $a$ runs over a certain arithmetic progression of modulus $k$ which divides $g_{0} m F$ where $m=N \mathfrak{m}$. Hence, we see that the inner sum in (6.23) can be arranged as the sum of $n$ sums, each of which runs over a single segment of an arithmetic progression of length $\ll x^{1 / n}$. Since $\chi_{\mathfrak{q}}(\ell)=(\ell / \mathfrak{q})$ is the real primitive Dirichlet character of modulus $q=N \mathfrak{q}$ (see (3.17) and Lemma 3.6), we have here $n$ incomplete character sums of length $\ll x^{1 / n}$ and modulus $q \ll x$. Therefore, Conjecture $C_{n}$ applies, (or rather its consequence for arithmetic progressions as described at the end of Section 9), giving (see (9.4)),

$$
T(x ; \beta) \ll g_{0} x^{\frac{1-\delta}{n}+\varepsilon}
$$

except when $q \mid k$. Note that $q \mid k$ implies $q \mid m$.

4. On page 729 , replace lines $4-6$ by

Recall that (6.25) holds if $q \nmid m$. Hence, because of the factorizations (6.18) and (6.21), we have (6.25) unless the following holds:

$$
p\left|N\left(\beta-\beta^{\prime}\right) \Rightarrow p^{2}\right| m F N\left(\beta-\beta^{\prime}\right) .
$$

5. Accordingly, in the four following displays, replace respectively:

$F^{2} N\left(\beta-\beta^{\prime}\right)$ squarefull by $p\left|N\left(\beta-\beta^{\prime}\right) \Rightarrow p^{2}\right| m F N\left(\beta-\beta^{\prime}\right)$,

$F^{2} N(\gamma)$ squarefull by $p\left|N(\gamma) \Rightarrow p^{2}\right| m F N(\gamma)$,

$F^{2} N \mathfrak{b}$ squarefull by $p\left|N \mathfrak{b} \Rightarrow p^{2}\right| m F N \mathfrak{b}$,

$F^{2} b$ squarefull by $p\left|b \Rightarrow p^{2}\right| m F b$,

and then, on the line preceding (6.27), before the words "Hence, we conclude", insert the sentence:

Note that we can assume $m \leq x$ else the sum in (6.1) is empty.

6. On page 730, change the third sentence after (6.33) to the following one:

... The condition for $N(\gamma)$ is now replaced by $g \mid N(\gamma)$.

7. The argument following (9.7) up to the end of Section 9 is correct, but no longer sufficient for the application. Therefore, we replace it by the following modification: 
Now, suppose that $\chi=\chi_{q}$ is real, primitive of odd modulus $q$. For $n=$ $\mathrm{km}+\ell$, since $q$ is necessarily squarefree, we have

$$
\chi(n)=\chi_{(q, k)}(\ell) \chi_{q /(q, k)}(k) \chi_{q /(q, k)}(m+r)
$$

with $k r \equiv \ell(\bmod q /(q, k))$. It follows that the sum (9.7) is bounded by $S_{\chi q /(q, k)}\left(M^{\prime}, N / k\right)$ with $M^{\prime}=(M-\ell) k^{-1}+r$. Since the bound (9.4) does not depend on $M$, it remains valid for $S_{\chi}(M, N ; k, \ell)$ provided $\chi(\bmod q)$ is real, primitive with $q$ odd, $q \nmid k$. 